\title{
A Study on Mining User-Aware Uncommon Consecutive Topic Patterns in Report Streams
}

\author{
Ankit Gururaj1)
}

\begin{abstract}
Textual documents created and distributed on the Internet are constantly changing in different structures. The greater part of existing works is given to subject demonstrating and the development of individual topics, while sequential relations of topics in progressive reports distributed by a particular client are disregarded. In this paper, keeping in mind the end goal to describe and identify customized and anomalous practices of Internet clients, we propose Sequential Topic Patterns (STPs) and figure the issue of mining User-mindful Rare Sequential Topic Patterns (URSTPs) in archive streams on the Internet. They are uncommon all in all however moderately visit for particular clients, so can be connected in some genuine situations, for example, real-time monitoring on abnormal user behaviors. We show a gathering of calculations to tackle this inventive mining issue through three stages: preprocessing to remove probabilistic themes and recognize sessions for various clients, producing all the STP applicants with (expected) bolster values for every client by example development, and selecting URSTPs by making client mindful irregularity investigation on determined STPs. Probes both genuine (Twitter) and manufactured datasets demonstrate that our approach can without a doubt find extraordinary clients and interpretable URSTPs successfully and effectively, which significantly reflect users' characteristics.
\end{abstract}

Keywords : sequential topic patterns, user-mindful rare sequential topic patterns, twitter, internet, datasets.

\section{Introduction}

Record streams are made and disseminated in various forms on the Internet, such as news streams, emails, micro-blog articles, chatting messages, research paper archives, web forum discussions, and so forth. The substance of these reports generally concentrates on some[1-5].

specific topics, which reflect offline social events and users' characteristics in real life. To mine these bits of data, a considerable measure of looks into of content mining concentrated on separating themes from report accumulations and record streams through different probabilistic point models, for example, established PLSI [6][15], LDA [7] and their expansions [8][16-19][24-26].

Exploiting these extracted topics in document streams, most of existing works analyzed the Received(September 5, 2016), Review Result(1st: September 23, 2016, 2nd: October 20, 2016), Accepted(December 10, 2016)

${ }^{1}$ (Corresponding Author) Vidyaa Vikas Educational Institutions, Mysore - Bannur Road, Alanahally, Mysuru, Karnataka, India

email: Ankit7359@gmail.com 


\section{A Study an Mining User-Aware Uncommon Consecutive Topic Patterns in Report Streams}

evolution of individual topics to detect and predict social events as well as user behaviors [8] [11][12][23]. Be that as it may, few inquires about focused on the relationships among various subjects showing up in progressive records distributed by a particular client, so some covered up yet huge data to uncover customized practices has been disregarded. Keeping in mind the end goal to describe client practices in distributed report streams, we contemplate on the connections among subjects removed from these records, particularly the consecutive relations, and determine them as Sequential Topic Patterns (STPs). Each of them records the total and rehashed conduct of a client when she is distributing a progression of reports, and are appropriate for construing clients' inherent qualities and mental statuses. Firstly, contrasted with individual themes, STPs catch both blends and requests of points, so can serve well as discriminative units of semantic relationship among archives in uncertain circumstances. Furthermore, contrasted with report based examples, point based examples contain conceptual data of archive substance and are along these lines helpful in bunching comparable records and discovering a few regularities about Internet clients. Thirdly, the probabilistic description of topics helps to maintain and accumulate the uncertainty degree of individual topics, and can thereby reach high confidence level in pattern matching for uncertain data.

\section{Proposed system}

\subsection{Related work}

Topic mining in document collections has been extensively studied in the literature. Topic Detection and Tracking (TDT) task [3], [9] expected to recognize and track points (occasions) in news streams with bunching construct methods in light of watchwords. Considering the co-event of words and their semantic affiliations, a great deal of probabilistic generative models for extricating themes from archives were additionally proposed, for example, PLSI [15], LDA [7] and their expansions coordinating diverse components of records [5], [19], [24], and also models for short messages[16].

In numerous genuine applications, document collections for the most part convey transient data and can along these lines be considered as record streams. Different element theme demonstrating strategies have been proposed to find points after some time in archive streams [6], [18] and afterward to foresee disconnected get-togethers [8], [11], [23]. In any case, these techniques were intended to build the advancement model of individual points from a report stream, as opposed to investigate the connections among numerous subjects extricated from 
progressive records for particular clients.

Sequential pattern mining is an important problem in data mining, and has also been well studied so far. With regards to deterministic information, a far reaching study can be found in [21], [25]. The concept support [25] is the most well known measure for assessing the recurrence of a successive example, and is characterized as the number or extent of information arrangements containing the example in the objective database. Many mining calculations have been proposed in light of support, for example, FreeSpan [13].

\subsection{Existing system}

Most of existing works analyzed the evolution of individual topics to recognize and anticipate social events as well as user behaviors.

Many mining algorithms have been proposed in view of support, for example, PrefixSpan, FreeSpanand SPADE. They found successive consecutive examples whose bolster qualities are at least a client characterized edge, and were reached out by SLPMiner to deal with length decreasing support constraints.

Muzammal et al. [26] focused on sequence-level uncertainty in consecutive databases, and proposed strategies to assess the recurrence of a successive example in view of expected support, in the frame of candidate generate-and-test or pattern-growth.

\subsection{Disadvantages of existing system}

The obtained patterns are not always interesting for our purpose, because those rare but significant patterns representing personalized and abnormal behaviors are pruned due to low supports.

Furthermore, the algorithms on deterministic databases are applicable for document streams, as they neglected to handle the vulnerability in subjects.

\subsection{Proposed system}

In request to describe user behaviors in published document streams, we study on the correlations among topics extracted from these reports, particularly the successive relations, and 


\section{A Study on Mining User-Aware Uncomman Consecutive Topic Patterns in Report Streams}

indicate them as Sequential Topic Patterns (STPs).

To solve the innovative and significant problem of mining URSTPs in record streams, numerous new specialized difficulties are raised and will be handled in this paper.

Firstly, the input of the task is a textual stream, so existing systems of successive example digging for probabilistic databases can't be specifically connected to solve this problem.

A preprocessing stage is vital and significant to get abstract and probabilistic depictions of records by subject extraction, and afterward to perceive finish and repeated activities of Internet users by session identification.

Secondly, view of the real-time requirements in many applications, both the accuracy and the efficiency of mining algorithms are essential and ought to be considered, particularly for the probability computation process.

Thirdly, different from frequent patterns, the user-aware rare pattern concerned here is a new concept and a formal measure must be very much characterized, with the goal that it can successfully portray the majority of customized and irregular practices of Internet clients, and can adjust to various application situations. What's more, correspondingly, unsupervised digging calculations for this sort of uncommon examples should be outlined in a way different from existing frequent pattern mining algorithms.

\subsection{Advantages of proposed system}

To the best of our insight, this is the principal work that gives formal definitions of STPs and their irregularity measures, and advances the issue of mining URSTPs in document streams, with a specific end goal to portray and recognize customized and strange practices of Internet clients.

We propose a system to logically take care of this issue, and configuration relating algorithms to support it.

At initially, we give preprocessing systems with heuristic techniques for point extraction and session recognizable proof. At that point, acquiring the thoughts of example development in unverifiable environment, two alternative algorithms are intended to find all the STP applicants with support values for each user. That provides a trade-off between accuracy and efficiency. Finally, we exhibit a client mindful irregularity examination calculation as indicated by the formally characterized model to choose URSTPs and related clients.

We approve our approach by conducting investigations on both genuine and manufactured datasets. 


\section{Conclusion and Future work}

Mining URSTPs in published document streams on the Internet is a significant and challenging problem. It defines another sort of complex occasion designs in light of archive points, and has wide potential application situations, for example, continuous checking on unusual practices of Internet clients. In this paper, a few new ideas and the mining issue are formally characterized, and a gathering of calculations are outlined and consolidated to efficiently take care of this issue. The investigations led on both genuine (Twitter) and manufactured datasets exhibit that the proposed approach is extremely powerful and effective in finding uncommon clients and fascinating and interpretable URSTPs from Internet document streams, which can well capture users' personalized and abnormal behaviors and characteristics.

As this paper advances a creative research heading on Web information mining, much work can be based on it later on. At to begin with, the issue and the approach can likewise be connected in different fields and situations. Particularly for perused archive streams, we can see perusers of records as customized clients and make setting mindful proposal for them. Likewise, we will refine the measures of user-aware irregularity to oblige distinctive necessities, enhance the mining calculations for the most part on the level of parallelism, and study on-the-fly calculations going for realtime archive streams. Additionally, in view of STPs, we will attempt to characterize more perplexing occasion examples, for example, forcing timing imperatives on successive points, and configuration comparing proficient mining algorithms. We are likewise inspired by the double issue, i.e., finding STPs happening habitually in general, yet moderately uncommon for particular clients. Besides, will build up some down to earth instruments for reallife tasks of user behavior analysis on the Internet.

\section{References}

[1] C. C. Aggarwal, Y. Li, J. Wang, and J. Wang, Frequent pattern mining with uncertain data, Proc. ACM SIGKDD’09, (2009), pp.29-38.

[2] R. Agrawal and R. Srikant, Mining sequential patterns, Proc. IEEE ICDE'95, (1995), pp.3-14.

[3] J. Allan, R. Papka, and V. Lavrenko, On-line new event detection and tracking, Proc. ACM SIGIR'98, (1998), pp.37-45.

[4] T. Bernecker, H.-P. Kriegel, M. Renz, F. Verhein, and A. Zuefle, Probabilistic frequent itemset mining in uncertain databases, Proc. ACM SIGKDD’09, (2009), pp.119-128. 
[5] D. Blei and J. Lafferty, Correlated topic models, Adv. Neural Inf. Process. Syst., (2006), Vol.18, pp.147-154.

[6] D. M. Blei and J. D. Lafferty, Dynamic topic models, Proc. ACM ICML'06, (2006), pp.113-120.

[7] D. Blei, A. Ng, and M. Jordan, Latent Dirichlet allocation, J. Mach. Learn. Res., (2003), Vol.3, pp.993-1022.

[8] J. Chae, D. Thom, H. Bosch, Y. Jang, R. Maciejewski, D. S. Ebert, and T. Ertl, Spatiotemporal social media analytics for abnormal event detection and examination using seasonal-trend decomposition, Proc. IEEE VAST'12, (2012), pp.143-152.

[9] K. Chen, L. Luesukprasert, and S. T. Chou, Hot topic extraction based on timeline analysis and multidimensional sentence modeling, IEEE Trans. Knowl. Data Eng., (2007), Vol.19, No.8, pp.1016-1025.

[10] C. K. Chui and B. Kao, A decremental approach for mining frequent itemsets from uncertain data, Proc. PAKDD’08, (2008), pp.64-75.

[11] W. Dou, X. Wang, D. Skau, W. Ribarsky, and M. X. Zhou, LeadLine: Interactive visual analysis of text data through event identification and exploration, Proc. IEEE VAST'12, (2012), pp.93-102.

[12] G. P. C. Fung, J. X. Yu, P. S. Yu, and H. Lu, Parameter free bursty events detection in text streams, Proc. VLDB'05, (2005), pp.181-192.

[13] J. Han, J. Pei, B. Mortazavi-Asl, Q. Chen, U. Dayal, and M. Hsu, FreeSpan: frequent pattern-projected sequential pattern mining, Proc. ACM SIGKDD’00, (2000), pp.355-359.

[14] N. Hariri, B. Mobasher, and R. Burke, Context-aware music recommendation based on latent topic sequential patterns, Proc. ACM RecSys'12, (2012), pp.131-138.

[15] T. Hofmann, Probabilistic latent semantic indexing, Proc. ACM SIGIR'99, (1999), pp.50-57.

[16] L. Hong and B. D. Davison, Empirical study of topic modeling in Twitter, Proc. ACM SOMA'10, (2010), pp.80-88.

[17] Z. Hu, H.Wang, J. Zhu, M. Li, Y. Qiao, and C. Deng, Discovery of rare sequential topic patterns in document stream, Proc. SIAM SDM'14, (2014), pp.533-541.

[18] A. Krause, J. Leskovec, and C. Guestrin, Data association for topic intensity tracking, Proc. ACM ICML'06, (2006), pp.497-504.

[19] W. Li and A. McCallum, Pachinko allocation: DAG-structured mixture models of topic correlations, Proc. ACM ICML'06, (2006), Vol.148, pp.577-584.

[20] Y. Li, J. Bailey, L. Kulik, and J. Pei, Mining probabilistic frequent spatio-temporal sequential patterns with gap constraints from uncertain databases, Proc. IEEE ICDM'13, (2013), pp.448-457.

[21] N. R. Mabroukeh and C. I. Ezeife, A taxonomy of sequential pattern mining algorithms, ACM Comput. Surv., (2010), Vol.43, No.1, pp.3:1 - 3:41.

[22] A. K. McCallum, MALLET: A machine learning for language toolkit, (2002), [Online]. Available: http://mallet.cs.umass.edu 
[23] Q. Mei, C. Liu, H. Su, and C. Zhai, A probabilistic approach to spatiotemporal theme pattern mining on weblogs, Proc. WWW'06, (2006), pp.533-542.

[24] D. Mimno, W. Li, and A. McCallum, Mixtures of hierarchical topics with Pachinko allocation, Proc. ACM ICML'07, (2007), pp.633-640.

[25] C. H. Mooney and J. F. Roddick, Sequential pattern mining - approaches and algorithms, ACM Comput. Surv., (2013), Vol.45, No.2, pp.19:1 - 19:39.

[26] M. Muzammal, Mining sequential patterns from probabilistic databases by pattern-growth, Proc. BNCOD'11, (2011), pp.118-127. 
A Study on Mining User-Aware Uncommon Consecutive Topic Patterns in Report Streams

(This page is empty intentionally) 